\title{
Touch-Free Finger Sensing using Laser Light for Keys and Keyboards
}

\author{
Dinesh K. Anvekar ${ }^{1}$, Shanthi M. B. ${ }^{2}$, Ohileshwari M.S. ${ }^{3}$, Sanchari Saha ${ }^{4}$, Smitha N. ${ }^{5}$, Sundaraguru R ${ }^{6}$, \\ Phanindar Ravi $^{7}$, and Fairooz R. Ali $^{8}$ \\ Dept. of ECE, RNS Institute of Technology, Bengaluru, Karnataka ${ }^{1,3,5}$, \\ Dept. of CSE, CMR Institute of Technology, Bengaluru, Karnataka ${ }^{2}$, \\ Dept. of ISE, MVJ College of Engineering, Bengaluru, Karnataka ${ }^{4}$ \\ Sir M. Visvesvaraya Institute of Technology, Bengaluru, Karnataka ${ }^{6,7}$ \\ dinesh.anvekar@gmail.com¹, shanthi.mb@cmrit.ac.in², ohileshwarims@gmail.com ${ }^{3}$, saha.sanchari85@gmail.com ${ }^{4}$, \\ smithnesara81@gmail.com ${ }^{5}$, sugursg@ sirmvit.edu ${ }^{6}$, Phaninder_ec@ sirmvit.edu ${ }^{7}$, rustamfairooz@yahoo.in ${ }^{8}$
}

\section{ABSTRACT}

Keys and consoles are used in various authentic connections, particularly human- machine interactions in the present state of art of Human Machine Interfaces (HMI). Automatic Teller Machines (ATMs), Charge/MasterCard machines, and pass-code-based support frameworks are a few cases wherein an individual has to touch the keys to provide a secret code are instructions to the machines. However, in situations such as the new COVID 19 pandemic, it is not desirable for customers to touch the same keys as it may cause easy spreading of chronic infections. There is therefore a need to provide a way to input data by users without physically touching any keys. This need is met in our design by offering a device and method to accomplish key or keyboard operation input operation based on the detection of a user finger with low power the laser light.

Keywords:

pass-code, HMI, ATM, frameworks, console, detecting

Article Received: 18 October 2020, Revised: 3 November 2020, Accepted: 24 December 2020

\section{INTRODUCTION}

Assistive-technologies have attracted the attention of several Human-Computer Interaction (HCI) researchers over the past few decades. The keyboard remains a key element in ensuring people to communicate and engage in everyday activities. As our communication technologies advance and we become increasingly dependent on websites and apps to facilitate our interpersonal interactions. Most of the computational devices make it mandatory for the user to input the user login credentials to be entered through the press of a single key or a series of keystrokes. These kinds of user inputs require the user to physically touch the keys on some keypad/keyboard for input of the required data. The physical touch of the keys may lead to possible spread of common infectious diseases. Recent outbreak of COVID-19 pandemic has created the requirement for the inventions in data communication technologies for developing the touch-free devices for data inputs by maintaining the hygiene in the surrounding environment. Couple of researchers have focused on implementing touch-free virtual keyboards designed for disabled people with tetra-paresis [1] wherein each key can be selected by three double eye blinks registered by an EMG sensor.

Adam Nowosielski et al. in [2] have implemented a touch-free keyboard specifically for the physically challenged people who are unable to operate standard electronic equipment or computer input devices. In this keyboard system, 'typing' is done with head movements which are captured by thermal sensors. In the research work [3], Sean Cronin et al. have used gestures and head movement for the input of values through keyboard. The main focus of this research was to enable touch-free interaction with computer equipment in the hospital environment. Most of research and development activities being 
currently carried out in this area consider the need of specific users with disabilities. The present situation of COVID-19 outbreak has made it necessary to touch-less input de vices even for normal persons for prevention of the spreading of the COVID-19 and maintain hygiene in public health. This paper presents a novel design for implementing a touch-free finger sensing keyboard using the laser technology for reading the user input.

\section{RELATED STUDY}

In October 1997, Thomas Jakobin developed a laser actuated keyboard device [12] that had a laser pointer mounted on an adjustable headband to direct a collimated beam to a laser keyboard identified by a set of photo-sensors to be illuminated by the user-target beam. To provide a comparatively retina-safe output, during the laser acquisition mode the laser pointer output is controlled whereas to provide a higher return, during the recorded signal mode the laser pointer out- put is controlled. The laser pointer is modulated at a selected frequency during the higher-power mode to assist the laser keyboard in neither discriminating against normal background light. When the beam illuminates a targeted area for a selected percentage of a pre-determined dwell period a successful key-press is confirmed.

In May 2010, Yung-Lung Liu and Ta Ya Shiang introduced a keyboard consisting of a laser pointer [13] to produce laser radiation and a micro-gyroscope to calculate rotational angular velocity or angular acceleration while the keyboard swings. The laser pointer contains a first switch placed on the keyboard, a laser device facing an aperture on the keyboard, a printed circuit board (PCB) behind the laser unit, and a rechargeable battery being electrically connected together. Ben Seewoster implemented a mechanism for inserting a code into an ATM keypad [12]. Housing that can be positioned over an ATM keypad is part of the innovative system. For working individual keys of the ATM keypad, a multitude of solenoids are fixed to the lower surface of the housing. For inserting a PIN number into a controller, a code entering key is placed on the upper surface of the housing such that a code can be inserted into the system within the privacy of a vehicle and subsequently transferred to the ATM key pad of a visible ATM machine.

Kurt D. Newman, $M$ et al., developed a complex Automatic Teller Machine (ATM) keypad device [13] to avoid attacking the PIN number of users by watching the hand motion to dial the PIN. Any time the user enters the PIN, the keyboard display for entering the PIN has been changed to avoid a potential attack based on the user's hand movement. Atul Gupta has developed a system [14] that generates a dynamic and spatial keyboard on an automated teller machine (ATM). This innovation entails obtaining ATM users' preferred language information and retrieving the numerical values, messages, and any other characters needed to be displayed on the dynamic keyboard. The dynamic keypad is created in the preferred language based on the retrieved information.

In Jan. 2010, Felix A. Monet al. created a software product [15] that enables users to interface on the basis of pre-established user interests with the auto- mated teller machine (ATM). An ATM is supported, for example, with: (1) a user interface designed to accept user identifying information from a user; and (2) a computing unit operationally connected to the user interface and configured to use user identifying information to determine a preestablished user preference. In 2019, Zirui Niu have introduced a password input keyboard [16] with a touch screen configured to receive a password input by a user's finger. A pass- word verifying module has been configured to determine whether the password received is the same as the one that stored at the bank database. An unlocking and controlling module configured to obtain determination results from the password 
verifying module and the fingerprint verifying module and operate a locked device that is associated with the password input keyboard.

Based on the literature study, till date, all the inventions have focused on making a secure and user-friendly keyboard for different operations for user authentication. The present pandemic situation due to COVID-19 has opened ample space for inventions to enable touch-free finger sensing in different applications.

\section{PROPOSED SYSTEM}

We have proposed a new approach to enable touch-free finger sensing with low power laser light for the purpose of entering the data through the keyboards in different daily applications like ATM transactions, Lift- operations and many more. This approach will create a safe environment in public places by preventing the spreading of the infections caused by viruses and germs. The proposed touch-free solution provides two levels of touch free sensing of key inputs by users.

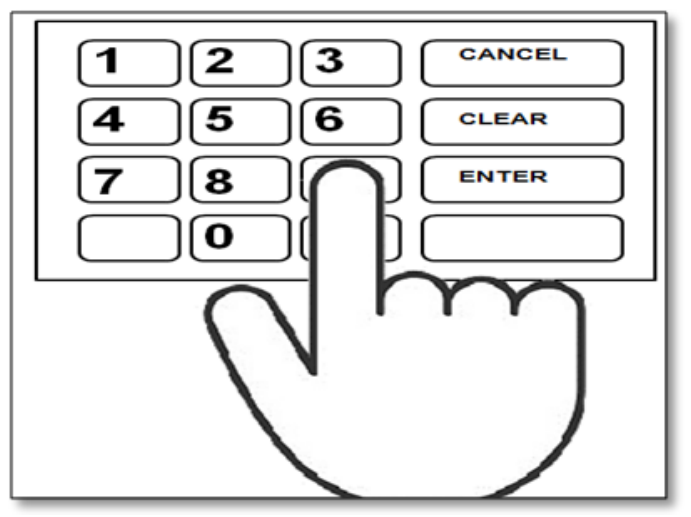

(a). A typical ATM keyboard

\subsection{Touch-free finger sensing for individual key}

Fig. 1 shows the finger sensing for a single key as compared to pressing of a key for a conventional key in a keyboard. As shown in Fig. 1a, an ATM keyboard allows a user to enter numbers and give of commands by pressing a key on it. The same function of pressing a key can be achieved in our proposed design by inserting a finger in the empty space within a cuboidal box structure without touching its sides or the base as shown in Fig. 1b). The cuboid has mirrored surfaces on two of its opposite sides. A low power laser source is mounted behind a mirrored side towards a corner. Behind the other mirrored side near the opposite corner a photo detector such as a light dependent resistor (LDR) or photodiode is mounted. The position and inclination of the laser source and the photo detector are so adjusted that the laser beam emanating from the laser source undergoes multiple reflections between the two mirrored surfaces and finally hits the photo detector.

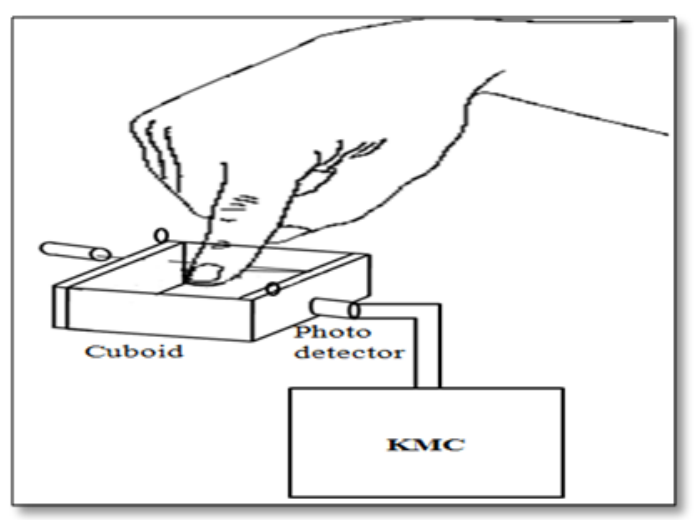

(b). Proposed Laser based touch-free key box

Fig. 1 Touch sensitive and Touch-free Keyboards

The terminals of the photo detector are connected is to a key monitoring circuit (KMC) by means of two wires. Thus, in the normal state, the photo detector produces a signal that indicates that the laser light from the laser source is detected implying that no finger has been inserted in the empty space within the key box. 
When an user inserts his finger in the space within the key box as shown in Fig. 1b, the laser beam passing to and from between the mirrored sides is obstructed by the finger and it does not reach the photo detector. This condition is sensed by the $\mathrm{KMC}$ as an assertion of the key by the user and sends a key assertion signal to another electronic system for performing the necessary action. As the finger does not come in physical contact with any part of the key box apparatus, spreading of germs and viruses among multiple users of the key apparatus is greatly reduced.

\subsection{Touch-free sensing for Keyboards}

The idea of sensing a finger with a laser source, photo detector, and mirrors for a single key function can be extended to multiple keys in a keyboard matrix. The Fig.2 illustrates the design of touch-free keyboard using laser.

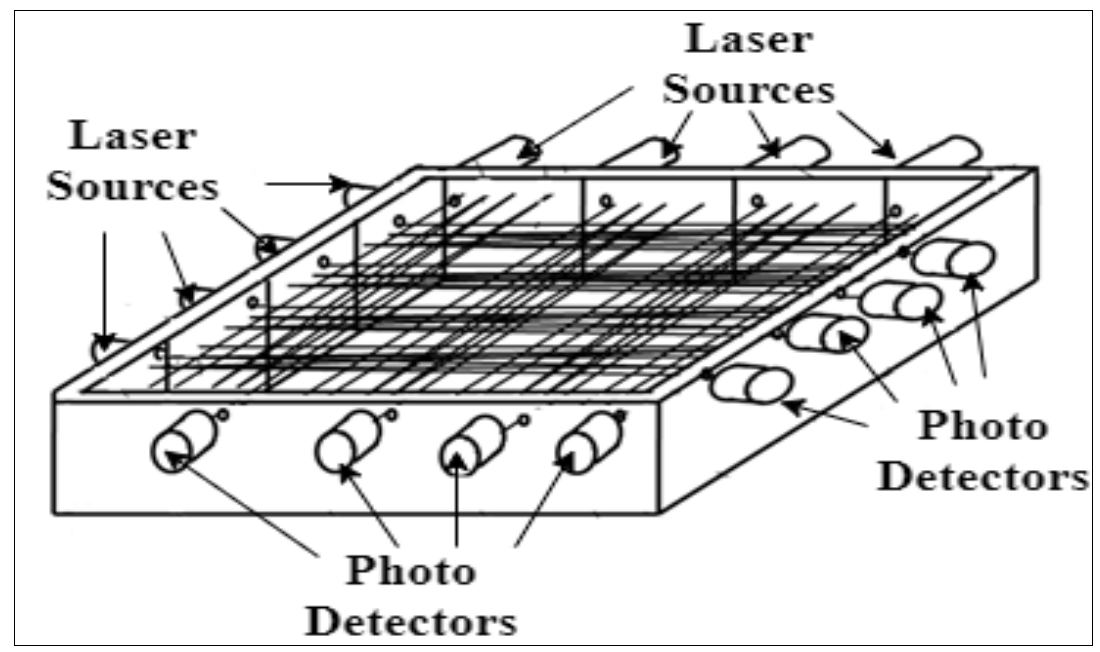

Fig. 2 Keyboard design for Touch-free sensing

Fig.2 shows a 4 x 4 keyboard matrix apparatus as per the current invention. Four laser sources are mounted on the outer side of and behind apertures of one face of a square frame and their corresponding photo detectors are mounted on the outer side of and behind apertures of the opposite face of the frame such that the laser beams from the laser sources hit the photo detectors, after repeated reflections from the inner mirrored. Similarly, The laser beams traversing back and forth between the mirrors form a 2 dimensional rectangular coverage area. The intersection of the 2 dimensional rectangular coverage areas from the 8 laser sources forms 16 square regions. A key monitoring circuit (KMC) connected to the photo detectors and by means of two wires each for each photo detector.

Fig. 3 shows a situation where in a user's finger is inserted into the intersecting square region of laser beams. As the finger intercepts the laser beams from laser sources, the laser beam does not reach the corresponding photo detectors. All the other six photo detectors receive the laser beams emanating from their corresponding laser sources.

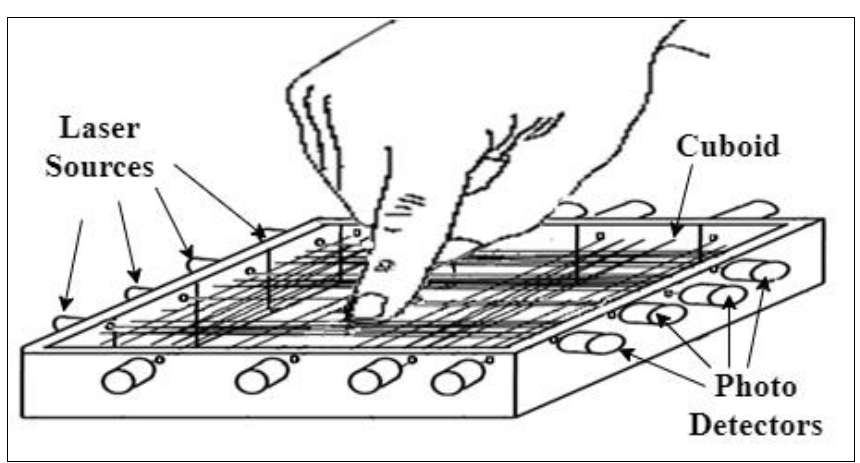

Fig. 3 Design of $4 \times 4$ Touch-free Laser Keyboard. 
This condition can be easily interpreted by electronic circuitry similar to the key monitoring circuit indicating that the key position has been asserted by the user. Thus, by using the key matrix apparatus the keyboard function of a 4 x 4 matrix keyboard can be achieved without a user's finger touching physically any part of the apparatus as per the objective of our invention.

\section{EXPERIMENTAL IMPLEMENTATION}

An experimental implementation of a single key box is shown in Fig. 4. When there is no presence of finger in the key box, the output of the LDR based light detector (KMC) is High and the output LED is ON as seen in Fig. 4a. When a user inserts his finger in the space within the key box as shown in Fig.4b, the laser beam passing to and

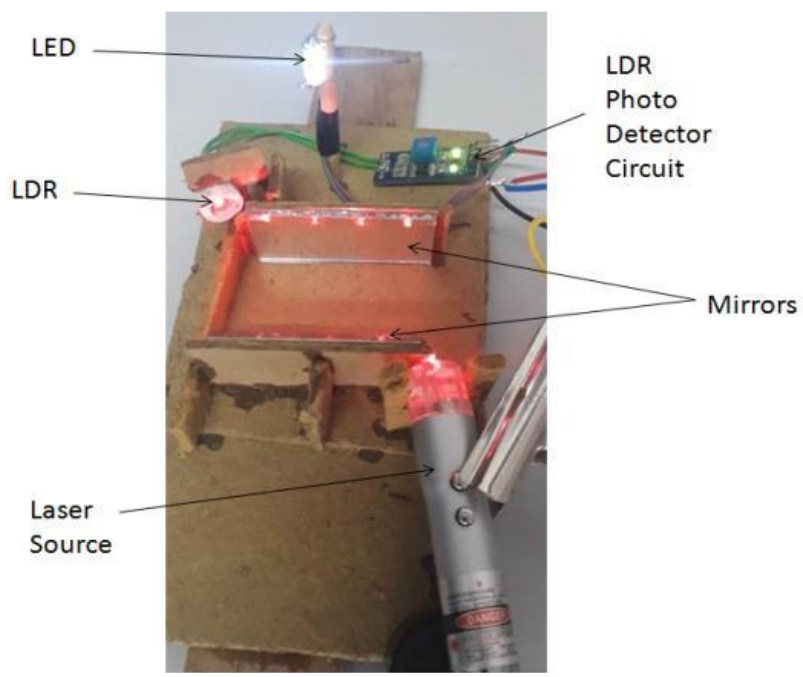

(a) Low output without finger (LED ON)

\section{CONCLUSION}

We have proposed a novel invention by designing a Touch-free finger sensing using the laser technology for enabling the user to enter the mandatory inputs for getting access to perform transactions using public gadgets. The present situation of COVID-19 pandemic has opened wide requirements for the inventions in the development of touch-free operations in while using the public gadgets for the transactions to prevent the spread of viral infections among the people who uses these devices. Through this from between the mirrored sides is obstructed, and hence does not reach the photo detector, which in our implementation is an LDR (Light Dependent Resistor). Consequently, the resistance of the LDR is high and the output LED is OFF. This condition is detected by the $\mathrm{KMC}$ as an assertion of the key by the user and this key assertion signal (digital active low output) is given to an electronic device to perform the required action. As the finger is not in physical contact with any part of the key box apparatus, this greatly reduces the spreading of germs and any virus among multiple users of the key apparatus. A similar type of implementation with 8 laser sources and 8 LDR based photo detectors can be used for detecting 16 keys in a key matrix.

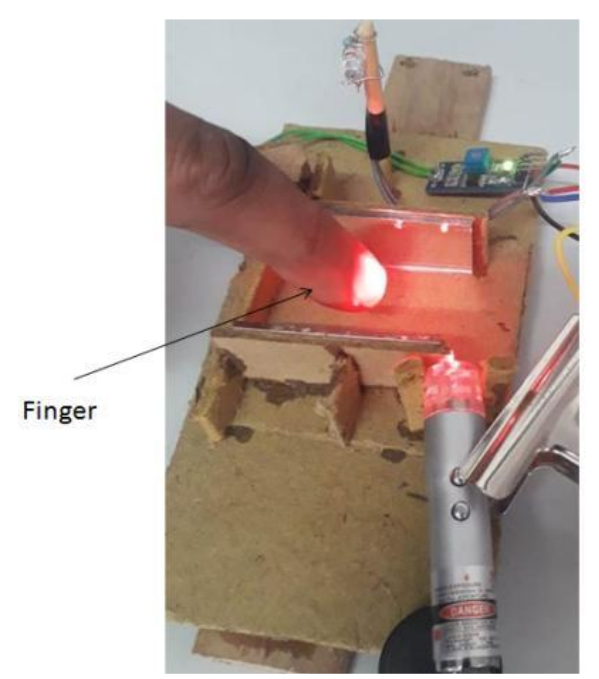

(b) High output with finger inserted in the key box (LED OFF)

research work, we have designed a touch-free finger sensing approach which could be used in the devices including single key press or having a keyboard to operate on, for supplying authentication information to get the access to the device. This would protect the user not only getting panic while taking the common public services in workplace or in public services and prevent the spread of harmful deceases. In future, this research can be considered for the massproduction of these devices which are the immediate requirement in near future. 


\section{REFERENCES:}

[1] Dobosz, K., \& Stawski, K. (2017). Touchless Virtual Keyboard Controlled by Eye Blinking and EEG Signals. Man-Machine Interactions 5, 5261. doi:10.1007/978-3-319-67792-7_6

[2] Adam Nowosielski and Pawel Forczmański, Touchless typing with head movements captured in thermal spectrum, Pattern Analysis and Applications (2019) 22:841-855, doi.org/10.1007/s10044-0180741-0.

[3] Shivam Rustagi, Aakash Garg, Pranay Raj Anand, Rajesh Kumar, Yaman Kumar and Rajiv Ratn Shah, Touchless Typing Using Head Movement-based Gestures, IEEE Sixth International Conference on Multimedia Big Data (BigMM),2020. 978$1-7281-9325-0 / 20 / \$ 31.00$ C2020 IEEE DOI 10.1109/BigMM50055.2020.00025

[4] Nowosielski A. (2018) 3-Steps Keyboard: Reduced Interaction Interface for Touchless Typing with Head Movements. In: Kurzynski M., Wozniak M., Burduk R. (eds) Proceedings of the 10th International Conference on Computer Recognition Systems CORES 2017. CORES 2017. Advances in Intelligent Systems and Computing, vol 578. pp. 229-237, DOI 10.1007/978-3-319-59162-9_24, Springer.

[5] Vidat Ganga, Saaima T, Swetha Kumari, Eye ball movement controlled mouse and keyboard using camera, International Journal of Advance Research, Ideas and Innovations in Technology, ISSN: 2454132X , Impact factor: 4.295 (Volume 5, Issue 2),2019.

[6] Sean Cronin, Gavin Doherty, Touchless computer interfaces in hospitals: A review, Health Informatics Journal 2019, Vol. 25(4) 1325-1342, The Author(s) 2018, www.psychologyandeducation.net
Article

reuse guidelines: sagepub.com/journals-permissions, DOI: $10.1177 / 1460458217748342$.

[7] C. Yu, Y. Gu, Z. Yang, X. Yi, H. Luo, and Y. Shi, "Tap, dwell or gesture? exploring head- ased text entry techniques for hmds," in CHI '17, 2017.

[8] A. Markussen, M. R. Jakobsen, and K. Hornbundefinedk, "Vulture: A mid-air word-gesture keyboard," in CHI '14, 2014.

[9] J. Tu, H. Tao, and T. Huang, "Face as mouse through visual face tracking," Comput. Vis. Image Underst., vol. 108, no. 1-2, p. 35-40, Oct. 2007.

[10] S. Zhai and P. O. Kristensson, "The wordgesture keyboard: Reimagining keyboard interaction," Commun. ACM, 2012.

[11] D. Shukla, R. Kumar, A. Serwadda, and V. V. Phoha, "Beware, your hands reveal your secrets!" ser. ACM CCS, 2014.

[12] https://patents.justia.com/patent/6097373

[13] https://patentimages.storage.googleapis.co m/ec/ac/84/5520d14316d64a/US20110279 287A1.pdf

[14] https://patents.justia.com/patent/5616900

[15] https://patentimages.storage.googleapis.co m/38/34/52/5894cab91314c6/US9196111. pdf

[16] https://patentimages.storage.googleapis.co $\mathrm{m} / \mathrm{a} 5 / \mathrm{cf} / \mathrm{d} 3 / 40 \mathrm{ffc} 58322 \mathrm{~b} 903 / \mathrm{US} 201400285$ 66A1.pdf 